\title{
ON A CONJECTURE OF ERDÖS ABOUT ADDITIVE FUNCTIONS UDC 519.21
}

\section{KARL-HEINZ INDLEKOFER}

Abstract. For a real-valued additive function $f: \mathbb{N} \rightarrow \mathbb{R}$ and for each $n \in \mathbb{N}$ we define a distribution function

$$
F_{n}(x):=\frac{1}{n} \#\{m \leq n: f(m) \leq x\} .
$$

In this paper we prove a conjecture of Erdös, which asserts that in order for the sequence $F_{n}$ to be (weakly) convergent, it is sufficient that there exist two numbers $a<b$ such that $\lim _{n \rightarrow \infty}\left(F_{n}(b)-F_{n}(a)\right)$ exists and is positive.

The proof is based upon the use of the Stone-Cech compactification $\beta \mathbb{N}$ of $\mathbb{N}$ to mimic the behavior of an additive function as a sum of independent random variables.

\section{INTRODUCTION}

A function $f: \mathbb{N} \rightarrow \mathbb{R}$ is called additive if $f(m n)=f(m)+f(n)$ for any coprime integers $m$ and $n$. Then $f$ is defined by its values $f\left(p^{k}\right)$ on prime powers $p^{k}$ ( $p$ prime, $k \in \mathbb{N}$ ) and $f(1)=0$.

Given a real-valued additive function $f$, one can define, for each $n \in \mathbb{N}$, a distribution function

$$
F_{n}(x):=\frac{1}{n} \#\{m \leq n: f(m) \leq x\} .
$$

An old conjecture of Erdös in 1947 (see Erdös 4]) asserts that in order for the sequence $F_{n}$ to be (weakly) convergent (in this case we say that the additive function $f$ possesses a limit distribution), it is sufficient that there exist two numbers $a<b$ such that

$$
\lim _{n \rightarrow \infty}\left(F_{n}(b)-F_{n}(a)\right) \text { exists and is positive. }
$$

In 1992 A. Hildebrand [6] showed that the conclusion of Erdös' conjecture is valid, provided (1.2) is strengthened to

$$
L_{a}:=\lim _{n \rightarrow \infty} F_{n}(a) \text { and } L_{b}:=\lim _{n \rightarrow \infty} F_{n}(b),
$$

both exists and $L_{a} \neq L_{b}$. Some further discussions are contained in [10] and [11].

In this paper we show that the above conjecture of Erdös holds.

Theorem. Let $f: \mathbb{N} \rightarrow \mathbb{R}$ be an additive function. In order for the distributions (1.1) to converge, it is sufficient that (1.2) holds for some $a<b$.

The proof is based upon a method, introduced in [7, 8] using the Stone-Čech compactification $\beta \mathbb{N}$ of $\mathbb{N}$ to mimic the behavior of an additive function as a sum of independent random variables.

2010 Mathematics Subject Classification. Primary 11N37, 11N60, 11K65.

Key words and phrases. Probabilistic number theory, additive functions. 


\section{Finitely Distributed AdDitive FunCtions}

An additive function $f$ is said to be finitely distributed if there are positive constants $c_{1}$ and $c_{2}$, and an unbounded sequence $n_{1}<n_{2}<\ldots$ so that for every $i$ there exists a sequence

$$
a_{1}^{(i)}<a_{2}^{(i)}<\cdots<a_{t_{i}}^{(i)}<n_{i}
$$

satisfying

$$
\left|f\left(a_{r}^{(i)}\right)-f\left(a_{s}^{(i)}\right)\right|<c_{1}, \quad t_{i}>c_{2} n_{i}, 1 \leq r, s \leq t_{i} .
$$

The necessary and sufficient condition that $f$ should be finitely distributed is that there should exist a constant $c$ and an additive function $h$ so that

$$
f(n)=c \log n+h(n)
$$

where both series

$$
\sum_{|h(p)|>1} \frac{1}{p}, \quad \sum_{\substack{p \\|h(p)| \leq 1}} \frac{h^{2}(p)}{p}
$$

converge (Erdös [3], 1946). Further characterizations of finitely distributed additive functions can be found in Chapter 7 of Elliott's book [2]. For our purpose we shall apply the following result ([2, p. 259]).

Proposition. If an additive function admits representation (2.1) with convergent series (2.2), then, if we define

$$
\alpha(n)=c \log n+\sum_{\substack{p \leq n \\|h(p)| \leq 1}} \frac{h(p)}{p}
$$

the distribution functions

$$
G_{n}(x):=\frac{1}{n} \#\{m \leq n: f(m)-\alpha(n) \leq x\}
$$

weakly converge to some distribution function $G(x)$.

If (1.2) holds, then $f$ is finitely distributed. Now, assume that (2.1) holds and $\alpha(n)$ is unbounded. Then, if $\alpha\left(n_{k}^{\prime}\right) \rightarrow \infty, k \rightarrow \infty$, for some subsequence $\left(n_{k}^{\prime}\right)$, then

$$
\lim _{k \rightarrow \infty}\left\{G_{n_{k}^{\prime}}\left(b-\alpha\left(n_{k}^{\prime}\right)\right)-G_{n_{k}^{\prime}}\left(a-\alpha\left(n_{k}^{\prime}\right)\right)\right\}=\lim _{k \rightarrow \infty}\left(F_{n_{k}^{\prime}}(b)-F_{n_{k}^{\prime}}(a)\right)>0
$$

by (1.2), whereas the left side in (2.5) tends to zero since $G_{n}$ converge weakly to some distribution function. Then, since $\alpha(n)=c \log n+O(\log \log n)$, we conclude $c=0$, i.e., $f=h$, and

$$
A(n):=\sum_{\substack{p \leq n \\|f(p)| \leq 1}} \frac{f(p)}{p}=O(1) \quad \text { for all } n \in \mathbb{N} .
$$

In the following we assume that

$$
\sum_{\substack{p \\|f(p)| \leq 1}} \frac{f(p)}{p} \text { diverges, }
$$

which implies (see [3], Theorem II) that $G(x)$ is continuous and strictly increasing for all $x \in \mathbb{R}$. 
For each $n \in \mathbb{N}$ define the additive function $f_{n}$ by

and put, for $A \subset \mathbb{N}$,

$$
f_{n}\left(p^{k}\right)= \begin{cases}f\left(p^{k}\right), & \text { if } p \leq n, \\ 0, & \text { otherwise }\end{cases}
$$

If the limit

$$
\delta_{n}(A):=\frac{1}{n} \#\{m \leq n: m \in A\}
$$

$$
\delta(A):=\lim _{n \rightarrow \infty} \delta_{n}(A)
$$

exists we say that $A$ possesses the asymptotic density $\delta(A)$.

If some sequence $\left\{n_{k}^{\prime}\right\}$ is given we write

$$
\delta^{\prime}(A):=\lim _{k \rightarrow \infty} \delta_{n_{k}^{\prime}}(A)
$$

in case the limit (2.9) exists.

With these notations we show

Lemma 1. Assume that (1.2) holds. Then

$$
\lim _{n \rightarrow \infty} \delta\left(\left\{m: f_{n}(m) \in(a, b]\right\}\right)=\delta(\{m: f(m) \in(a, b]\})=: c_{0}>0 .
$$

Proof. Observe that $\delta\left(\left\{m: f_{n}(m) \in(a, b]\right\}\right)$ always exists. Assume that (2.10) does not hold. Then there exists a sequence $\left\{n_{k}\right\}$ of natural numbers such that

$$
\lim _{k \rightarrow \infty} \delta\left(\left\{m: f_{n_{k}}(m) \in(a, b]\right\}\right)=c^{\prime} \neq c_{0} .
$$

Since $A\left(n_{k}\right)=O(1)$ there exists some subsequence $\left\{n_{k}^{\prime}\right\}$ of $\left\{n_{k}\right\}$ so that

$$
\lim _{k \rightarrow \infty} A\left(n_{k}^{\prime}\right)=: A^{\prime}
$$

exists. Choose $k_{1}$ such that for every $k_{0} \geq k_{1}$,

$$
\begin{aligned}
& \left|\delta\left(\left\{m: f_{n_{k_{0}}}(m) \in(a, b]\right\}\right)-c_{0}\right| \\
& \quad=\left|\delta^{\prime}\left(\left\{m: f_{n_{k_{0}}^{\prime}}(m) \in(a, b]\right\}\right)-\delta^{\prime}(\{m: f(m) \in(a, b]\})\right| \\
& \quad \geq \frac{\left|c_{0}-c^{\prime}\right|}{2} .
\end{aligned}
$$

On the other hand, we shall show that

$$
\lim _{k_{0} \rightarrow \infty} \varlimsup_{k \rightarrow \infty} \delta_{n_{k}^{\prime}}\left(\left\{m:\left|f(m)-f_{n_{k_{0}}^{\prime}}(m)\right|>\varepsilon\right\}\right)=0
$$

for every $\varepsilon>0$ which contradicts (2.11).

For the proof of (2.12) put

$$
\mathcal{P}_{0}:=\{p:|f(p)|>1\} \cup\left\{p^{k}: k \geq 2\right\} .
$$

Define the functions

and

$$
h^{\prime}(m):=\sum_{\substack{p^{k} \| m \\ p^{k} \in \mathcal{P}_{0} \\ p>n_{k_{0}}^{\prime}}} f\left(p^{k}\right)
$$

$$
j(m):=\sum_{\substack{p \| m \\|f(p)| \leq 1 \\ p>n_{k_{0}}^{\prime}}} f(p) .
$$


We deduce from the definitions of these functions that

$$
f(m)-f_{n_{k_{0}}^{\prime}}(m)=j(m)-\left(A\left(n_{k}^{\prime}\right)-A\left(n_{k_{0}}^{\prime}\right)\right)+h^{\prime}(m)+\left(A\left(n_{k}^{\prime}\right)-A\left(n_{k_{0}}^{\prime}\right)\right) .
$$

We shall prove that for every $\varepsilon>0$ each of the three expressions

and

$$
\begin{gathered}
L_{1}\left(k_{0}\right)=\varlimsup_{k \rightarrow \infty} \delta_{n_{k}^{\prime}}\left(\left\{m:\left|j(m)-\left(A\left(n_{k}^{\prime}\right)-A\left(n_{k_{0}}^{\prime}\right)\right)\right|>\varepsilon\right\}\right), \\
L_{2}\left(k_{0}\right)=\varlimsup_{k \rightarrow \infty} \delta_{n_{k}^{\prime}}\left(\left\{m:\left|h^{\prime}(m)\right|>\varepsilon\right\}\right)
\end{gathered}
$$

$$
L_{3}\left(k_{0}\right)=\varlimsup_{k \rightarrow \infty} \delta_{n_{k}^{\prime}}\left(\left\{m:\left|A\left(n_{k}^{\prime}\right)-A\left(n_{k_{0}}^{\prime}\right)\right|>\varepsilon\right\}\right)
$$

converge to zero as $k_{0} \rightarrow \infty$. We may readily estimate the first of these three expressions by appealing to the Turán-Kubilius inequality. In our present circumstances it becomes

$$
\frac{1}{n_{k}^{\prime}} \sum_{m=1}^{n_{k}^{\prime}}\left|j(m)-\left(A\left(n_{k}^{\prime}\right)-A\left(n_{k_{0}}^{\prime}\right)\right)\right|^{2} \ll \sum_{\substack{n_{k_{0}}^{\prime}<p \leq n_{k}^{\prime} \\|f(p)| \leq 1}} \frac{|f(p)|^{2}}{p} .
$$

Appealing to the convergence of the second sum in (2.2) we see that

$$
L_{1}\left(k_{0}\right) \ll \frac{1}{\varepsilon^{2}} \sum_{\substack{n_{k_{0}}^{\prime}<p \\|f(p)| \leq 1}} \frac{|f(p)|^{2}}{p}=o(1) \quad \text { as } k_{0} \rightarrow \infty .
$$

The estimate $L_{3}\left(k_{0}\right)=o(1)$ as $k_{0} \rightarrow \infty$ is obvious.

If an integer $m$ is counted in the expression $L_{2}\left(k_{0}\right)$ it must satisfy one of two divisibility criteria.

First, it may be divisible by the square of a prime $p>n_{k_{0}}^{\prime}$. The frequency of these integers is at most

$$
\delta_{n_{k}^{\prime}}\left(\left\{m: p^{2} \mid m, p>n_{k_{0}}^{\prime}\right\}\right) \leq \sum_{n_{k_{0}}^{\prime}<p} \frac{1}{p^{2}}=o(1) \quad \text { as } k_{0} \rightarrow \infty .
$$

Next, it may be exactly divisible by a prime in the range $n_{k_{0}}^{\prime}<p$ for which $|f(p)|>1$. From the hypothesis (2.2) we deduce that the frequencies of such integers is at most

$$
\sum_{\substack{n_{k_{0}}^{\prime}<p \\|f(p)|>1}} \frac{1}{p}=o(1) \quad \text { as } k_{0} \rightarrow \infty
$$

and thus $L_{2}\left(k_{0}\right)=o(1)$ as $k_{0} \rightarrow \infty$. We have now shown that (2.12) holds and the proof of Lemma 1 is completed.

In the next step we identify the additive function $f$ with a sum $\sum_{p \text { prime }} X_{p}$ of independent random variables.

\section{AdDitive Functions as a SUM of INDEPENDENT RANDOM VARIABLES}

For the sake of simplicity we restrict ourselves to strongly additive functions. Then $f$ can be written in the form

$$
f=\sum_{p} f(p) \varepsilon_{p}
$$

where

$$
\varepsilon_{p}(n)= \begin{cases}1, & \text { if } p \mid n \\ 0, & \text { otherwise }\end{cases}
$$


If $\mathcal{A}$ denotes the algebra generated by the sets

$$
A_{p}:=\{n \in \mathbb{N}: p \mid n\}, \quad p \text { prime, }
$$

then obviously each $A \in \mathcal{A}$ possesses an asymptotic density $\delta(A)$ and $\delta\left(A_{p}\right)=\frac{1}{p}(p$ prime). Thus $\delta$ defines a content on $\mathcal{A}$. Now the construction runs as follows. (For details see $[7,8$.) We embed $\mathbb{N}$, endowed with the discrete topology, in the Stone-Cech compactification $\beta \mathbb{N}$,

$$
\mathbb{N} \hookrightarrow \beta \mathbb{N}
$$

and, if for any $A \subset \mathbb{N}$, the closure of $A$ in $\beta \mathbb{N}$ is denoted by $\bar{A}$, then

$$
\overline{\mathcal{A}}:=\{\bar{A} \subset \beta \mathbb{N}: A \in \mathcal{A}\}
$$

is an algebra, too. The extension $\bar{\delta}$ of $\delta$,

$$
\bar{\delta}(\bar{A}):=\delta(A), \quad \bar{A} \in \overline{\mathcal{A}},
$$

defines a premeasure on $\overline{\mathcal{A}}$ and leads to a measure $\mathrm{P}$, induced by

$$
\delta^{*}(A):=\varlimsup_{n \rightarrow \infty} \delta_{n}(A) \text { for all } A \subset \mathbb{N},
$$

and to a probability space $(\Omega, \sigma(\overline{\mathcal{A}}), \mathrm{P})$ with $\Omega=\beta \mathbb{N}$ and with $\mathrm{P}\left(\overline{A_{p}}\right)=1 / p, p$ prime.

There is a unique extension of $\varepsilon_{p}$ to a function $\bar{\varepsilon}_{p}$ on $\Omega$, and putting $X_{p}=f(p) \bar{\varepsilon}_{p}$,

$$
f=\sum_{p} f(p) \varepsilon_{p} \rightarrow X=\sum_{p} f(p) \bar{\varepsilon}_{p}=\sum_{p} X_{p}
$$

we get

$$
f_{n} \rightarrow S_{n}:=\sum_{p \leq n} X_{p}
$$

with

$$
\mathrm{P}\left(X_{p}=f(p)\right)=\frac{1}{p}
$$

and

$$
\mathrm{P}\left(X_{p}=0\right)=1-\frac{1}{p}
$$

The $\overline{\varepsilon_{p}}$ are independent, i.e., $X=\sum_{p} X_{p}$ is a sum of independent random variables. If (1.2) holds then, by Lemma 1 ,

$$
\lim _{n \rightarrow \infty} \mathrm{P}\left(S_{n} \in(a, b]\right)=c_{0}>0
$$

and, by Proposition above, the series $\sum_{p} X_{p}$ is essentially convergent (for the definition see [13, p. 262]).

Now writing

$$
a_{p}=\mathrm{E}\left(X_{p}^{c}\right), \quad Y_{p}=X_{p}-a_{p}, \quad T_{n}:=\sum_{p \leq n} Y_{p}
$$

then $\lim _{n \rightarrow \infty} T_{n}$ exists almost surely. (Here $X_{p}^{c}$ denotes the truncation of $X_{p}$ at (a positive) $c$, i.e., we replace $X_{p}$ by $X_{p}=X$ or 0 according as $\left|X_{p}\right|<c$ or $\left|X_{p}\right| \geq c$.) Denote $Y:=\lim T_{n}$ a.s.

It is well known that the a.s. convergence of $Y=\sum_{p} Y_{p}$ is equivalent to the weak convergence of the distributions of the partial sums of that series. Moreover, by Kolmogorov's three series theorem, $Y=\sum_{p} Y_{p}$ converges a.s. if and only if the series

$$
\sum_{p} \mathrm{E}\left(Y_{p}^{c}\right), \quad \sum_{p} \mathrm{P}\left(\left|Y_{p}\right|>c\right), \quad \sum_{p} \operatorname{Var}\left(Y_{p}^{c}\right)
$$

converge. 
We choose $c=1$, i.e., $a_{p}=\mathrm{E}\left(X_{p}^{1}\right)$ and put (see (2.6) $)$

$$
A(n)=\sum_{p \leq n} a_{p}
$$

Then $A(n)=O(1)$ and, the divergence of the sequence $A(n)$ implies (see [3, Theorem 2]).

Lemma 2. Let $Y=\sum_{p} Y_{p}$ with $Y_{p}=X_{p}-a_{p}$ as above, where the partial sums $\sum_{p \leq N} a_{p}$ are bounded and divergent. Then the distribution function $G(x)=\mathrm{P}(Y \leq x)$ is continuous and strictly monotone for all $x \in \mathbb{R}$.

Remark. The divergence of the sequence $A(n)$ implies

$$
\begin{aligned}
& \sum_{p} a_{p}^{-}=-\infty, \\
& \sum_{p} a_{p}^{+}=+\infty,
\end{aligned}
$$

where $a_{p}^{+}=\max \left(a_{p}, 0\right)$ and $a_{p}^{-}=\max \left(-a_{p}, 0\right)$. Then the strict monotonicity of the distribution function $G(x)$ in Lemma 2 can be directly proved by a result of A. Hildebrand [6].

For this we define, following the notation of Hildebrand in [6, p. 1206], the range of a random variable $X$ as the set

$$
R(X)=\{x \in \mathbb{R}: \mathrm{P}(|X-x| \leq \varepsilon)>0 \text { for every } \varepsilon>0\},
$$

that is, it is equal to the set of points of increase of the distribution function

$$
F(x)=\mathrm{P}(X \leq x) .
$$

The form of this set was described by A. Hildebrand in Lemma 2 of [6] when $X$ is given as an a.s. convergent series of independent random variables. A special version of this result is contained in the following lemma.

Lemma 3. Let $\sum_{n=0}^{\infty} X_{n}$ be an a.s. convergent series of independent random variables and let $X$ denote its sum. Suppose that for every $\varepsilon>0$ and $n \geq n_{0}=n_{0}(\varepsilon)$ there exist numbers $c_{n}^{-}=c_{n}^{-}(\varepsilon), c_{n}^{+}=c_{n}^{+}(\varepsilon) \in R\left(X_{n}\right)$ with $\left|c_{n}^{-}\right| \leq \varepsilon$ and $\left|c_{n}^{+}\right| \leq \varepsilon$ such that

$$
\lim _{N \rightarrow \infty} \sum_{n=n_{0}}^{N} c_{n}^{-}=-\infty
$$

and

$$
\lim _{N \rightarrow \infty} \sum_{n=n_{0}}^{N} c_{n}^{+}=+\infty .
$$

Then $R(X)=\mathbb{R}$.

Now it is easy to prove the assertions of Lemma 2. Put

$$
c_{p}^{-}= \begin{cases}f(p)-a_{p}, & \text { if }-\frac{\varepsilon}{2} \leq f(p)<0, \\ 0, & \text { otherwise }\end{cases}
$$

and

$$
c_{p}^{+}= \begin{cases}f(p)-a_{p}, & \text { if } 0<f(p) \leq \frac{\varepsilon}{2} \\ 0, & \text { otherwise }\end{cases}
$$


Then obviously, $c_{p}^{-}, c_{p}^{+} \in R\left(Y_{p}\right),\left|c_{p}^{-}\right| \leq \frac{\varepsilon}{2}+\left|a_{p}\right| \leq \varepsilon$ and $\left|c_{p}^{+}\right| \leq \frac{\varepsilon}{2}+\left|a_{p}\right| \leq \varepsilon$ for $p>n_{0}=n_{0}(\varepsilon)$ since $\left|a_{p}\right| \leq 1 / p$. Further,

$$
\begin{aligned}
\sum_{n_{0} \leq p \leq N} c_{p}^{-} & =\sum_{\substack{n_{0} \leq p \leq N \\
-\frac{\varepsilon}{2}<f(p)<0}} f(p)-\sum_{n_{0} \leq p \leq N} a_{p} \\
< & \sum_{\substack{n_{0} \leq p \leq N \\
-\frac{\varepsilon}{2}<f(p)<0}} \frac{f(p)}{p}+O(1) \\
< & \sum_{\substack{n_{0} \leq p \leq N \\
-1<f(p)<0}} \frac{f(p)}{p}+O(1) \\
= & \sum_{\substack{n_{0} \leq p \leq N\\
}} a_{p}^{-}+O(1) \rightarrow-\infty \quad \text { as } N \rightarrow \infty
\end{aligned}
$$

Here the last inequality holds because of the convergence of the second series in (3.1).

Similarly,

$$
\lim _{N \rightarrow \infty} \sum_{n_{0} \leq p \leq N} c_{p}^{+}=+\infty
$$

We use Lemma 3 and recall that the divergence of series (2.7) implies, by Lévy's theorem, the continuity of $G(x)$ to end the proof of Lemma 2 .

This ends the remark.

For every subsequence $n^{\prime}=\left(n_{k}^{\prime}\right)$ of natural numbers we define

$$
\delta^{\prime}(A)=\lim _{k \rightarrow \infty} \delta_{n_{k}^{\prime}}(A)
$$

if the limit exists. This leads to a content $\delta^{\prime}$ on $\mathcal{A}$ and a measure $P^{\prime}$ on $\beta \mathbb{N}$ induced by

$$
\delta^{\prime}(A)=\varlimsup_{k \rightarrow \infty} \delta_{n_{k}^{\prime}}(A) \text { for all } A \subset \mathbb{N} .
$$

Obviously, if $\Omega_{0} \subset \beta \mathbb{N}$ is $\mathrm{P}$-measurable it is $P^{\prime}$-measurable and $\mathrm{P}\left(\Omega_{0}\right)=P^{\prime}\left(\Omega_{0}\right)$.

Since every bounded real-valued function $g$ on $\mathbb{N}$ extends uniquely to a (continuous) function $\bar{g}$ on $\beta \mathbb{N}$ (for details see R. Walker [14, p. 8 et seq.]), we conclude

$$
\Omega_{0}:=\overline{\{m: f(m) \in(a, b]\}}=\{\omega: \bar{f}(\omega) \in[a, b]\},
$$

where $\bar{f}$ is the unique extension of the (bounded) function $f_{(a, b]}$, defined by

$$
f_{(a, b]}(m)= \begin{cases}f(m), & \text { if } f(m) \in(a, b], \\ |a|+|b|+1, & \text { if } f(m) \notin(a, b] .\end{cases}
$$

If (1.2) holds, then

$$
\mathrm{P}\left(\Omega_{0}\right)=c_{0}>0
$$

\section{Proof of the Conjecture of Erdös}

We suppose that $A(n)$ is not convergent so that

$$
\underline{A}:=\liminf _{n \rightarrow \infty} A(n)<\limsup _{n \rightarrow \infty} A(n)=: \bar{A},
$$

and we shall show that this leads to a contradiction.

We fix two increasing sequences $n^{\prime}=\left\{n_{k}^{\prime}\right\}$ and $n^{\prime \prime}=\left\{n_{k}^{\prime \prime}\right\}$ of positive integers so that

$$
\underline{A}=\lim _{k \rightarrow \infty} A\left(n_{k}^{\prime \prime}\right) \quad \text { and } \quad \bar{A}=\lim _{k \rightarrow \infty} A\left(n_{k}^{\prime}\right) .
$$


We put

$$
g_{n}=\sum_{p \leq n}\left(f(p) \varepsilon_{p}-a_{p}\right)
$$

and define

$$
g^{\prime}=g_{n_{1}^{\prime}}+\sum_{k=1}^{\infty}\left(g_{n_{k+1}^{\prime}}-g_{n_{k}^{\prime}}\right)
$$

Then

$$
\left\{m: g^{\prime}(m) \in(a-\bar{A}, b-\bar{A}]\right\}=\{m: f(m) \in(a, b]\}
$$

since $g^{\prime}(m)=f(m)-\bar{A}$ for every $m \in \mathbb{N}$. Further,

$$
\delta^{\prime}\left(\left\{m: g^{\prime}(m) \in(a-\bar{A}, b-\bar{A}]\right\}\right)=\lim _{k \rightarrow \infty} \delta^{\prime}\left(\left\{m: g_{n_{k}^{\prime}}(m) \in(a-\bar{A}, b-\bar{A}]\right\}\right)=c_{0} .
$$

In the same way we define

$$
g^{\prime \prime}=g_{n_{1}^{\prime \prime}}+\sum_{k=1}^{\infty}\left(g_{n_{k+1}^{\prime \prime}}-g_{n_{k}^{\prime \prime}}\right)
$$

with $g^{\prime \prime}(m)=f(m)-\underline{A}, m \in \mathbb{N}$, and obtain

$$
\delta^{\prime \prime}\left(\left\{m: g^{\prime \prime}(m) \in(a-\underline{A}, b-\underline{A}]\right\}\right)=\lim _{k \rightarrow \infty} \delta^{\prime \prime}\left(\left\{m: g_{n_{k}^{\prime \prime}}(m) \in(a-\underline{A}, b-\underline{A}]\right\}\right)=c_{0} .
$$

Defining the corresponding extensions $\overline{g^{\prime}}$ and $\overline{g^{\prime \prime}}$ and $P^{\prime}$ and $P^{\prime \prime}$, respectively, we arrive at

$$
\Omega_{0}=\left\{\omega: \overline{g^{\prime}}(\omega) \in[a-\bar{A}, b-\bar{A}]\right\}=\left\{\omega: \overline{g^{\prime \prime}}(\omega) \in[a-\underline{A}, b-\underline{A}]\right\}
$$

together with

$$
P^{\prime}\left(\left\{\omega: \overline{g^{\prime}}(\omega) \in[a-\bar{A}, b-\bar{A}]\right\}\right)=P^{\prime \prime}\left(\left\{\omega: \overline{g^{\prime \prime}}(\omega) \in[a-\underline{A}, b-\underline{A}]\right\}\right)=c_{0} .
$$

Since

and since

$$
\begin{aligned}
& g^{\prime} \text { corresponds to } \quad Y^{\prime}=\lim _{k \rightarrow \infty} T_{n_{k}^{\prime}}, \\
& g^{\prime \prime} \text { corresponds to } \quad Y^{\prime \prime}=\lim _{k \rightarrow \infty} T_{n_{k}^{\prime \prime}}
\end{aligned}
$$

$$
Y=\sum_{p} Y_{p}=\lim _{n \rightarrow \infty} T_{n}
$$

converges a.s. with respect to $\mathrm{P}$ and possesses an everywhere continuous distribution function we conclude

(i) $\left\{\omega: Y^{\prime}(\omega) \in[a-\bar{A}, b-\bar{A}]\right\}=\Omega_{0}^{\prime}$ with $P^{\prime}\left(\Omega_{0} \Delta \Omega_{0}^{\prime}\right)=0$,

(ii) $P^{\prime}\left(\left\{\omega: Y^{\prime}(\omega) \in[a-\bar{A}, a-\underline{\underline{A}}]\right\}\right) \leq P^{\prime}\left(\left\{\omega: Y^{\prime}(\omega) \neq Y^{\prime \prime}(\omega)\right\}\right)=0$ and

(iii) $P^{\prime}\left(\left\{\omega: Y^{\prime}(\omega) \in[a-\underline{A}, b-\bar{A}]\right\}\right)=c_{0}$.

Observe, that (iii) implies that

$$
a-\underline{A}<b-\bar{A}
$$

Since $\mathrm{P}(\{\omega: Y(\omega) \in[a-\bar{A}, a-\underline{A}]\})$ exists it must be zero by (ii), i.e.,

$$
\mathrm{P}(\{\omega: Y(\omega) \in[a-\bar{A}, a-\underline{A}]\})=0 .
$$

In the same way we show

$$
\mathrm{P}(\{\omega: Y(\omega) \in[b-\bar{A}, b-\underline{A}]\})=0 .
$$

Equalities (4.3) and (4.4) contradict the monotonicity of $G(x)$, and thus the assertion of Theorem 1 holds. 


\section{ACKNOWLEDGEMENTS}

I would like to acknowledge and express my gratitude to my colleagues and friends Imre Kátai, Oleg Klesov and Robert Wagner for fruitful discussions and helpful comments.

\section{BIBLIOGRAPHY}

1. P. D. T. A. Elliott, A conjecture of Kátai, Acta Arith. XXVI (1974), 11-20. MR0354599 (50:7077)

2. P. D. T. A. Elliott, Probabilistic Number Theory I/II, Springer, New York, 1978/1980. MR551361 (82h:10002a)

3. P. Erdös, On the distribution function of additive functions, Ann. Math. 47 (1946), 1-20. MR0015424(7:416c)

4. P. Erdös, Some remarks and corrections to one of my papers, Bull. Amer. Math. Soc. 53 (1947), 761-763. MR0021030 (9:12e)

5. A. Hildebrand, Additive and multiplicative functions on shifted primes, Proc. London Math. Soc. 59(2) (1989), 209-232. MR1004429 (90i:11107)

6. A. Hildebrand, A note on the convergence of sums of independent random variables, Ann. Probab. 20 (1992), 1204-1212. MR1175258 (93g:60047)

7. K.-H. Indlekofer, A new method in probabilistic number theory, Probab. Theory Appl., Math. Appl. 80 (1992), 299-308. MR.1211915 (94e:11091)

8. K.-H. Indlekofer, New approach to probabilistic number theory - compactifications and integration, Adv. Studies Pure Math. 49 (2005), 133-170. MR2405602(2009d:11119)

9. I. Kátai, On the distribution of arithmetical functions on the set prime plus one, Composito Math. 19 (1968), 278-289. MR0244181 (39:5498)

10. F. Kong and Q. Tang, A theorem on the convergence of sums of independent random variables, Math. Acta Sci. 21 B (2001), 331-338. MR.1849532(2002f:60052)

11. F. Kong and Q. Tang, Notes on Erdös' conjecture, Math. Acta Sci. 20(4) (2000), 533-541. MR.1805022 (2001k:60032)

12. P. Lévy, Sur les séries dont les termes sont des variables éventuellement indépendents, Studia Math. 3 (1931), 119-155.

13. M. Loève, Probability theory I, Springer, Heidelberg-New York, 1977. MR0651017 (58:31324a)

14. R. Walker, The Stone-C̆ech Compactification, Springer, Heidelberg-New York, 1974. MR0380698 (52:1595)

Department of Mathematics, University of Paderborn, Warburger Strasse 100, 33098 PADERBorn, GERMANY

E-mail address: k-heinz@math.upb.de

Received 29/JAN/2013

Originally published in English 